\title{
Pulsed laser irradiation of a nanoparticles sensitised RDX crystal
}

\author{
Xiao Fang*, Matthew Stone and Christopher Stennett \\ Centre for Defence Chemistry, Cranfield University, Defence Academy of the \\ UK, Shrivenham SN6 8LA, UK.
}

\section{*Corresponding author. Email: x.fang@cranfield.ac.uk}

\begin{abstract}
The laser initiation of secondary explosives presents tactical, safety and environmental advantages over traditional ignition systems utilising primary explosives. In this paper, direct ignition or initiation by pulsed laser was investigated of cyclotrimethylenetrinitramine (RDX) crystals doped with gold nanoparticles (GNPs) for the first time. A nano-seconds pulsed laser was used for direct irradiation of the crystals at the GNP's surface-plasmon-resonance wavelength of $532 \mathrm{~nm}$. RDX crystals were recrystallised from a solution containing GNPs and characterised by visual microscopy for surface coating and subsurface doping. A high-speed camera was used to observe and capture the modes of interaction between the GNPs-doped RDX crystals and pulsed laser irradiation with a new classification method. Laser fluence thresholds for ignition and initiation were established. The GNPs sensitized RDX to pulsed laser irradiation at this selected wavelength is 3 orders of magnitude more sensitive than pure RDX. Direct initiation of the GNPs doped RDX crystal at laser fluence of $0.04 \mathrm{~J} / \mathrm{cm}^{2}$ led to the total consumption of a crystal sample, which was unreproducible with pure RDX. The results show that GNPs are effective optical sensitizers for direct initiation of RDX crystal by low-power pulsed laser.
\end{abstract}

Keywords: Gold nanoparticles; laser ignition; pulsed laser; explosive

\section{Introduction}

Current advances in explosives and ordnance are focused on achieving munitions that are safer to use, can be used and stored in a wide range of natural and artificial operating environments and which are environmentally safer to manufacture and demilitarise. Laser irradiation is a promising approach [1] to designing inherently safer munitions by eliminating high sensitivity primary explosives which are used in most of explosive initiation system. One of the limitations of current laser ignition systems is the ignition delay.; Many high precision applications such as high-velocity missiles cannot tolerate milliseconds delay when using a continuous wave (CW) laser for igniting lowsensitive or insensitive secondary explosives [2]. However, the use of pulsed laser may reduce this delay and achieve a rapid ignition in such applications.

The ability of pulsed laser to ignite explosives by direct irradiation depends on the optical absorption of the material at the laser wavelength, as absorption may encourage photo-dissociation as well as thermal effects. Absorption also 
dictates the depth to which the laser can penetrate. While secondary explosives such as RDX are generally much more absorptive at wavelengths in the UV region, several energetic materials, including RDX, have narrow absorption peaks in the visible and infrared regions that occur at very specific wavelengths $[3,4]$. Research [5-7] demonstrated that secondary explosive RDX was ignitable with a UV laser (e.g. $355 \mathrm{~nm}$ ) of high pulse energy density (e.g. $\left.2.3-5.5 \mathrm{Jcm}^{-2}\right)$ but not with an IR laser (e.g. $1064 \mathrm{~nm}$ ). Therefore, direct laser ignition of pure secondary explosives needs a high laser power which demands bulky and costly laser devices. Optical sensitisation by using additives in insensitive explosives has been an efficient method to increase their optical absorption and enhance needed laser ignitibility. This can be achieved by doping light absorbing substances, commonly carbon black (CB). Such optical sensitisation have beendemonstrated for insensitive secondary explosives like 1,1-diamino2,2-dinitroethene (FOX-7), Hexanitrostilbene (HNS) and RDX mixed with small amount (up to $5 \mathrm{wt} \%$ ) of carbon black [2,8]. However, carbon black absorbs at a broad range of spectrum; its mixtures with explosives are also optically sensitised at undesired light wavelengths, lacking wavelength selectivity. The mixtures may not retain uniformity during manufacturing, storage and transportation, affecting their reliability and ignitibility consistency.

A novel method of optical sensitisation has recently been studied, where gold nanoparticles (GNPs) are embedded within the crystals of secondary explosives with good chemical compatibility. The study concluded that GNPs-embedded RDX crystals could be ignited by a low-power $\mathrm{CW}$ diode laser at the wavelength (e.g. $808 \mathrm{~nm}$ ) of GNP's surface plasmon resonance (SPR), while pure RDX crystals were unable to ignite $[9,10]$. For this mechanism, GNPs under laser irradiation absorb laser energy due to their SPR and typically heat up. The heat transfers from the GNP surface to the surrounding material with hotspots formed. These hotspots cause and accelerate exothermic decomposition of the surrounding explosive, leading to ignition when they reach at the ignition temperature of the explosive. The hotspots model is the dominant mechanism responsible for direct laser ignition [11-14].

This paper focuses on investigating the modes of direct interaction between GNPs sensitised RDX crystal and a pulsed laser. It aims at characterising the modes of interaction, analysing the severity thereof and comparing its interaction thresholds with pure RDX and CB-coated RDX.

\section{Experimental}

\subsection{Sample preparation}

Three types of crystal samples of RDX were prepared using re-crystallisation: pure RDX crystals (S1), $0.5 \mathrm{wt} \% \mathrm{CB}$ coated RDX crystals (S2) and $0.5 \mathrm{wt} \%$ GNP doped RDX crystals (S3). The method is detailed below.

S1 - Pure RDX: $600 \mathrm{mg}$ of RDX was weighed out and placed in a $30 \mathrm{ml}$ vial. 12 $\mathrm{ml}$ of analytical grade acetone (Sigma-Aldrich Ltd, UK) was added to dissolve the RDX at room temperature and a further $2 \mathrm{ml}$ of acetone were added to 
ensure the RDX fully dissolved and a slightly under-saturated solution was created. The solution was then transferred into a $60 \mathrm{ml}$ glass flask and sealed using a nylon film with three pin-holes pierced. The solution was left to evaporate naturally. When the sample was fully crystallised and dry it was ready for experimentation.

S2 - Carbon black coated RDX: using the same process as before, but this time adding $3 \mathrm{mg}$ of $\mathrm{CB}$ to the fully dissolved RDX acetone solution, the RDX crystals coated with $0.5 \mathrm{wt} \%$ concentration of carbon black was obtained and ready for use.

S3 - GNPs doped RDX: using the same process as for sample S2, but replacing the CB with carboxyl $(\mathrm{COOH})$ functionalized GNPs (NanoHybrids, USA), the RDX crystals doped with $0.5 \mathrm{wt} \%$ concentration of GNPs was obtained and ready for use. The $\mathrm{COOH}$ functional group was present to provide the affinity of GNPs to RDX, allowing the GNPs to remain embedded within or attached to the RDX crystals as they form. The GNPs were $60 \mathrm{~nm}$ diameter spheres with a SPR wavelength of $535 \mathrm{~nm}$ (Figure 1), closely matched to the used laser of $532 \mathrm{~nm}$ to give maximum optical density.
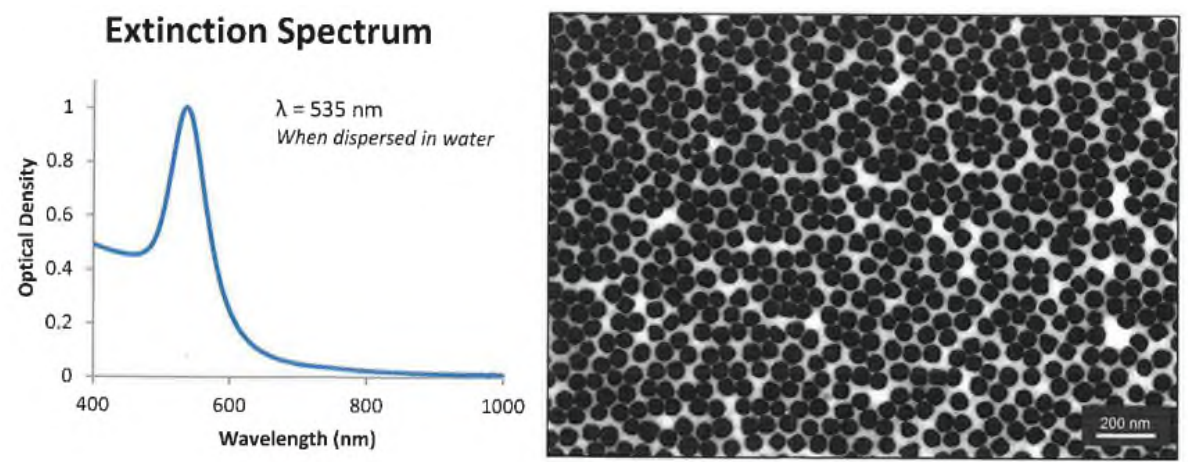

Figure 1. Extinction spectrum showing peak optical density at $535 \mathrm{~nm}$ (left), and SEM image of $60 \mathrm{~nm}$ GNP spheres (right).

All three batches (S1, S2 and S3) took approximately 20 days to fully crystallise and dry, as shown in Figure 2 with a standard ruler for scale. S1 was optically translucent. The appearance, shape and distribution of size were very similar between batches S1 and S3. S2 was opaque and presented only a few of single crystals larger than $1 \mathrm{~mm}$; the rest were a fine agglomeration of dust. S3 crystals were gold in colour and contained many "clumps" of small crystals which had joined together, leaving very few whole crystal samples.

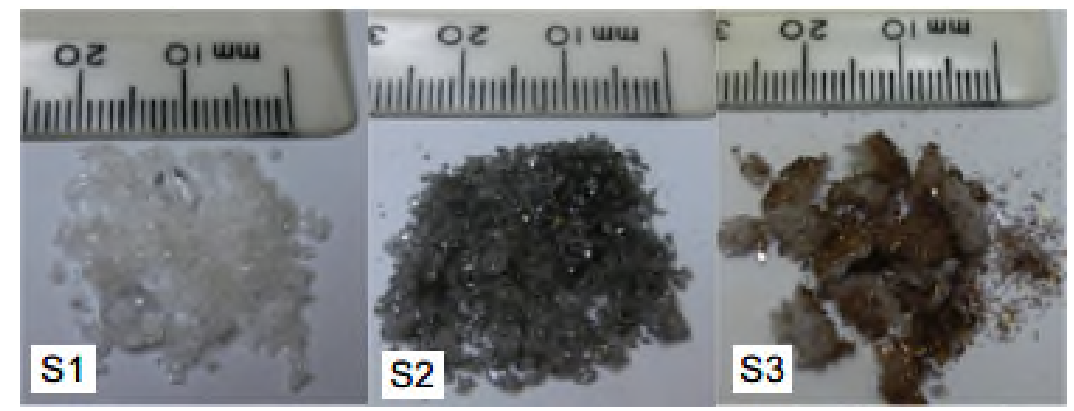


Figure 2. Photos of samples with a ruler for scale: $S 1$ (pure RDX): optically translucent, S2 (CB coated RDX): opaque and S3 (GNPs doped RDX): visible gold in colour.

\subsection{Sample characterisation}

Optical microscopy was conducted on representative crystals chosen from all 3 samples in order to characterise the crystals and understand whether subsurface doping or surface coating of the optical sensitiser was evident. Microscopy was conducted with a Leica DMLM microscope at up to $1000 \mathrm{x}$ magnifications in the front-lit mode of operation, with a Qimaging QiCam Fast 1394 camera for image capture. Crystals from each sample were selected and images were taken at low $50 \times$ magnification, which were then compared to images of a graticule to obtain a representative batch size. The higher levels of magnification were used to examine both the top and bottom surfaces of a sample and capture the images for analysis.

In order to determine if the optical sensitiser was present within the sample crystals, an etching process was applied to crystals of S2 and S3 to remove the surface layers before their microscopic work. To etch the crystals, a fine filter paper (Whatman grade 6, pore size $3 \mu \mathrm{m}$ ) was placed on a flattened glass plate and one drop of acetone was dispensed from a pipette onto the filter paper. The sample crystal was then slowly and gently rubbed in a figure-of-eight motion across the acetone-soaked paper while held with a plastic tweezer. This was maintained until enough surface depth was etched away, at which point the final etching was done in spaced lines, each on a fresh piece of filter paper so as to avoid self-contamination. This process was repeated on the reverse side in order to obtain "slices" of crystal, free from surface coating to be presented for microscopy.

\subsection{Experimental setup}

The experimental setup for laser irradiation is shown in Figure 3. The $1064 \mathrm{~nm}$ pulsed laser with 6 ns (Litron Laser, UK) pulse duration is frequency-doubled to obtain a $532 \mathrm{~nm}$ laser output. This laser beam is reflected by a prism and focused by a lens onto the sample placed on a glass plate as the sample holder to minimise the holder's interaction with the laser. The laser beam size of 1.2 $\mathrm{mm}$ in diameter was chosen to cover a sample and it was measured over laser marked spots in a sensitive paper using a Vernier microscope. The samples were then irradiated by the laser pulse at energy fluence of up to $\sim 9 \mathrm{~J} / \mathrm{cm}^{2}$. A digitising oscilloscope (Agilent Technologies DSO1024A) connected with the light detector (photodiode, Centronics Model no. BPX65) and a high-speed camera (Casio Exilim HS) operating at 1000 frames per second were used to measure and record the ignition process. A focusing lens $(50 \mathrm{~mm}$ in diameter and $50 \mathrm{~mm}$ in focal length) in front of the camera was used to magnify the image for the camera. 


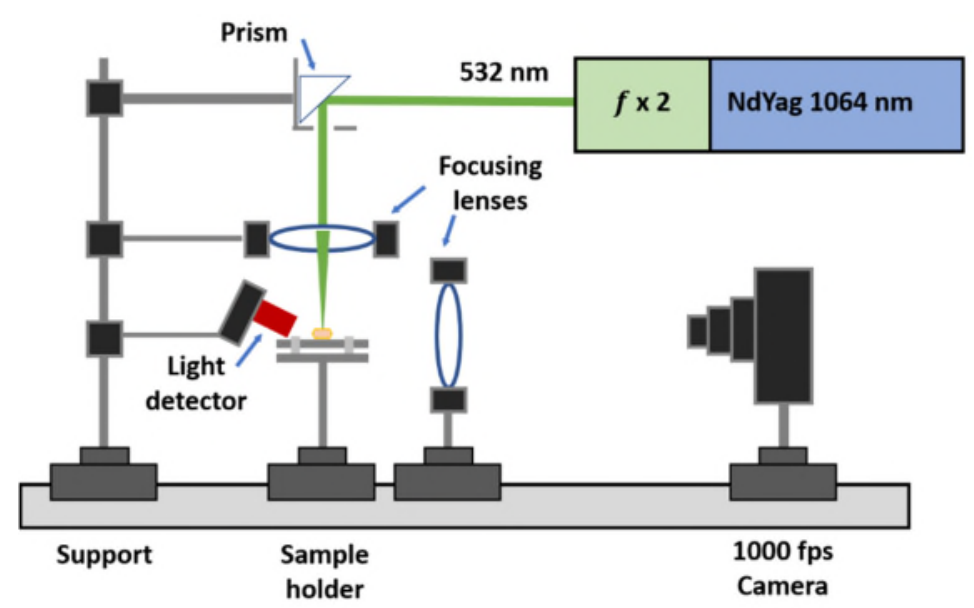

Figure 3. Laser ignition setup.

\section{Results and Discussion}

\subsection{Microscopy characterisation}

Carbon black doped samples: Microscopy of sample S2 confirmed carbon black presence on all surfaces examined. Concentration was greater on crystal/crystal boundaries and surface defects. There were also slightly higher concentrations of carbon black on the crystal surfaces which had been facing upwards. This was most likely due to top layer deposition on horizontal surfaces during evaporation. The rest of the surfaces had uniform coatings across them, as shown in Figure 4a-c. Post etching sample S2 showed no evidence of carbon black on the newly etched crystal surface or within the structure as shown in Figure $d$-f. It can, therefore, be concluded that as the RDX crystals formed, the carbon black was pushed out and displaced coating only the surfaces.

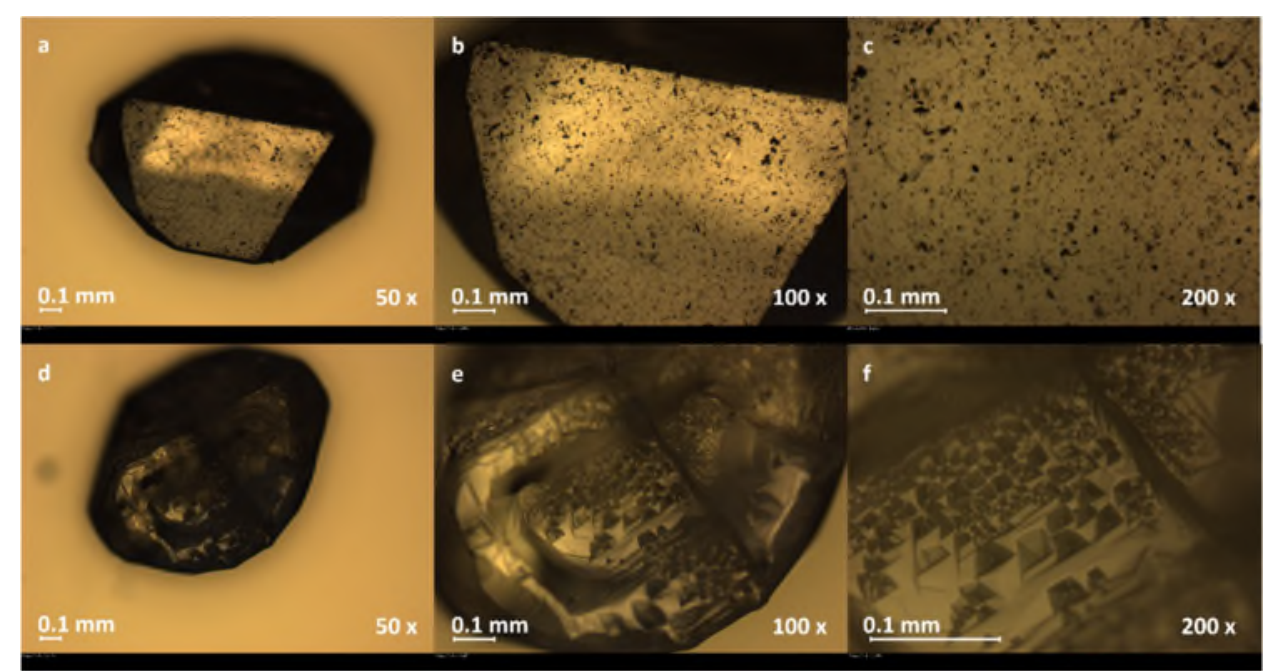

Figure 4. SEM images of CB-coated RDX at three magnifications, 50x, 100x and 200x: (a-c) the sample surface before etching, where visible specks of black clearly indicate that CB coating is present. (d-f) the same sample surface after etching, where no black specks are present, indicating CB is only doped on the sample surface. The images are differently orientated (e.g. between 
Figure $4 \mathrm{a}$ and $4 \mathrm{~d}$ ) due to the positioning of the pre- and post etching sample under the microscope.

GNP doped samples: GNP surface coating was evident and confirmed at each level of magnification as shown in Figure 5a-c. Large areas of high coating concentration such as those in Figure $5 \mathrm{~b}$ were common and pronounced on all crystals. They were more prevalent on the top side but still occurred on the bottom sides. These were consistent with pooling and deposition on the horizontal surface during the evaporation of the solution. The image in higher magnification (Figure 5c) revealed a consistent and even distribution of GNPs across the whole crystal surface. A thorough examination of the samples revealed this was true for all surfaces and crystals tested. It is possible that the $\mathrm{COOH}-\mathrm{PEGylated}$ GNPs did not covalently bond to the RDX and thus weren't doped within the structure, but that a weak interaction between the charges on the carboxylic acid and the RDX attracted them to the nucleation of the RDX crystals as they formed in the acetone. Post-etching sample S3 showed no evidence of GNPs at low magnifications (Figure 5d-e). However, at higher magnification (Figure 5f) a fine homogeneously dispersed layer of GNPs was evident in the sample. Smaller crystals showed larger amounts of GNPs on etched surfaces, probably due to faster crystal formation trapping GNPs within the crystal bodies or subsurface, and the distribution of their subsurface GNPs was very consistent.

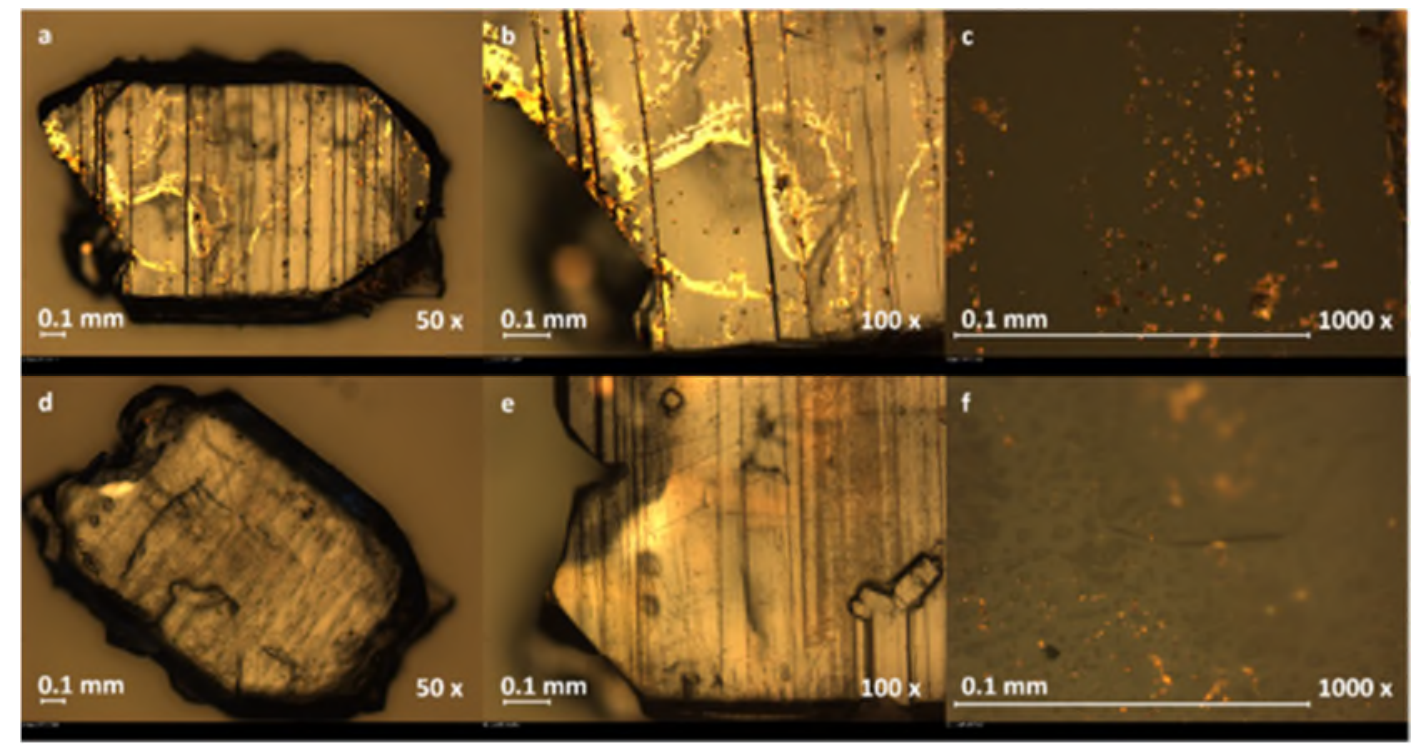

Figure 5. SEM images of GNPs doped RDX at three magnifications, 50x, 100x and 1000x: (a-c) the sample surface before etching; a) large surface GNP coatings, b) close-up of high concentration surface deposit, c) a high magnification closeup of the central area of $b$ ) which had a low concentration of surface coating; the image shows GNPs are still present and form a consistent fine coating. (d-f) the same sample surface after etching; d) and e) large surface deposits removed by etching, f) GNPs remaining in the etched surface, suggesting GNPs doping in the crystal. 


\subsection{Laser irradiation}

The tests of laser irradiation were conducted on all three samples to understand how GNPs doping affected modes of interaction between laser and samples, the delay and threshold for ignition and initiation and overall ignitability.

\subsubsection{Laser-induced modes of interaction}

Based on the observation of the sample response when it was subjected to laser irradiation, as shown in Figure 6, the mode of interaction of laser with the crystal samples can be classified as no (nil) interaction, ignition and initiation. They are described below.

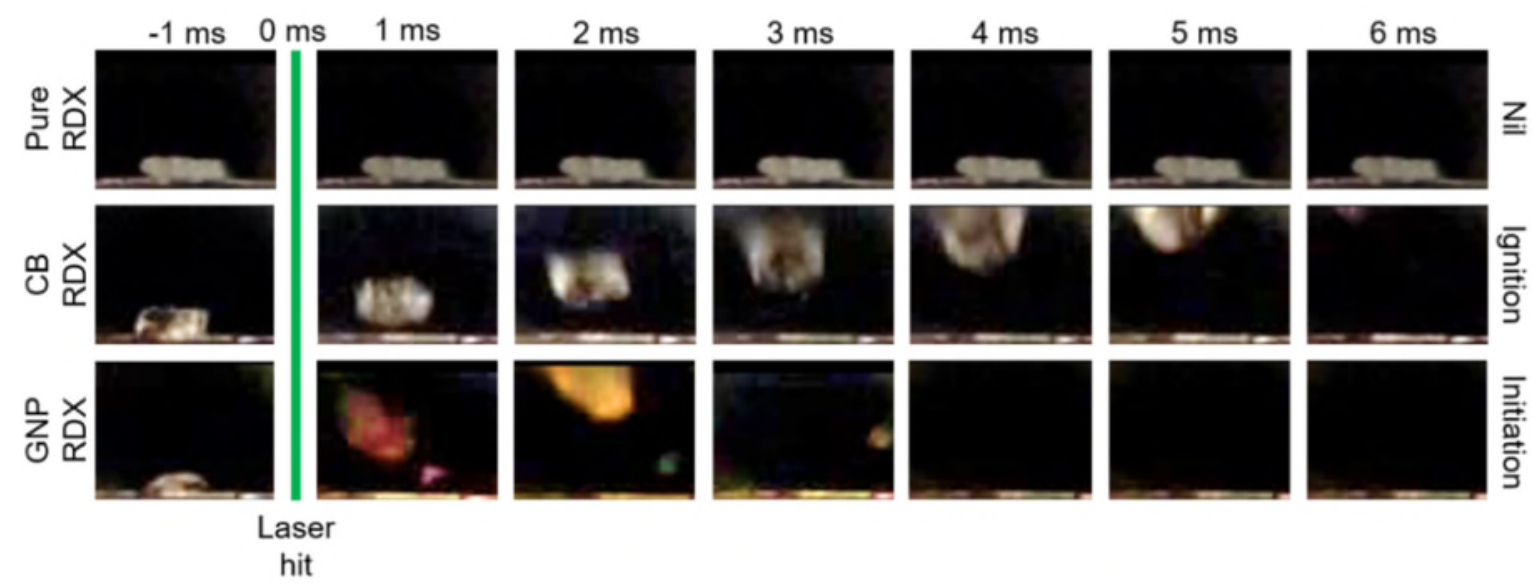

Figure 6. Three modes of interaction between RDX crystal samples and 532nm pulsed laser at an energy fluence of $1.4 \mathrm{~J} / \mathrm{cm}^{2}$, for pure RDX (nil interaction), CB coated RDX (igniting) and GNPs doped RDX (initiating).

No interaction: The sample was undamaged and unchanged during and after exposure to the pulsed laser irradiation. No sample movement was observed and no significant damage was observed at sample impact site on an investigation by visual microscopy.

Ignition: The ignition criteria are different from that of standard ignition; it includes the ablation of material by the pulsed laser causing plasma fireballs, micro-explosions at the surface or damage that consumed small part (up to $10 \%$ ) of sample mass. During the laser ignition firings, both plasma fireballs and micro-explosions were observed within $1 \mathrm{~ms}$ after laser irradiation. Plasma fireballs were clearly evident and captured by the high-speed camera, as shown in Figure 7a for three separate samples. Micro-explosions were audible as a sharp, short crack and were accompanied by movement of the sample on the sample holder. Figure $7 \mathrm{~b}$ shows three different samples moving in different directions, propelled by micro explosions at different speeds.

Initiation: The initiation criteria were defined as a significant portion of the sample being consumed as result of the pulsed laser irradiation or a runaway reaction initiated by the laser. Initiation either started with or was accompanied by a large plasma fireball. As shown in Figure 7c, three different samples were deemed to have initiated within $1 \mathrm{~ms}$ after being irradiated with the pulsed laser. 
The initiations were in all cases accompanied by plasma-fireballs and microexplosion. Upon close examination, stills from the high-speed video footage of samples that initiated showed a significant change in size or shape due to the sample being largely consumed; or initiated samples weren't recovered when they were fully burnt.

a) Plasma fireball

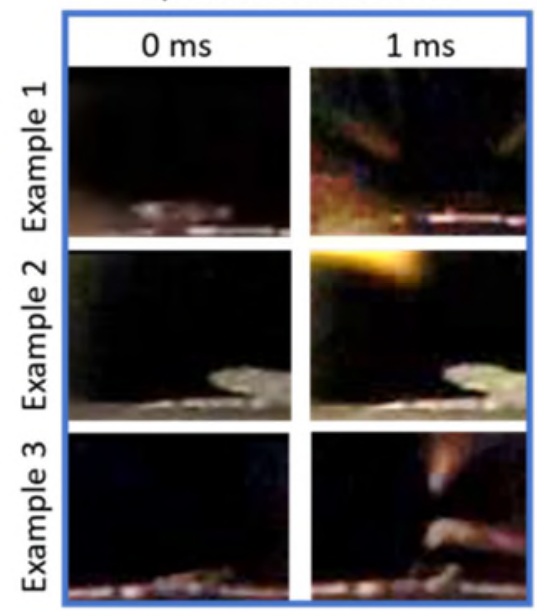

b) Micro explosion

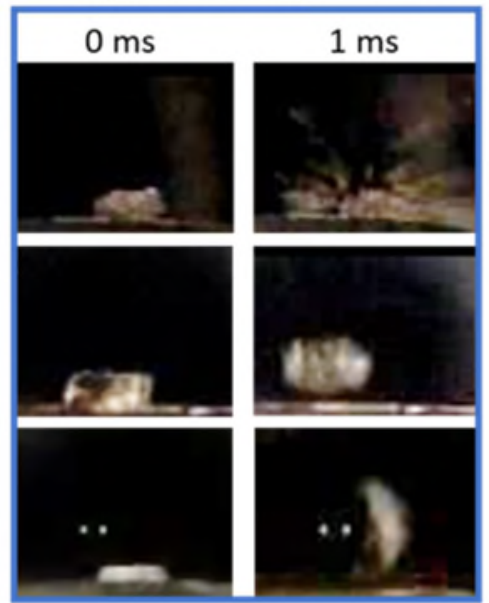

c) Initiation

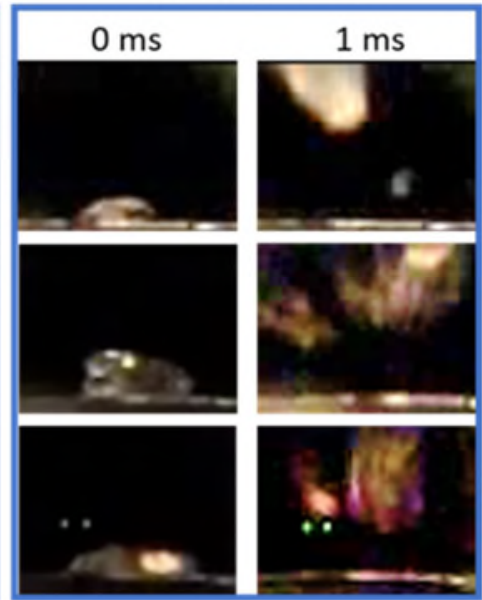

Figure 7. Examples of interactions between optically sensitised RDX crystals and 532nm pulsed laser, showing a) plasma fireballs, b) micro-explosions and c) initiation.

\subsubsection{Laser effect on modes of interaction}

To facilitate the comparative analysis of laser effect on the modes of interaction, ignition and initiation, the severity of the response of a sample in each mode of interaction was classified as 4 levels, from Nil to Violent for ignition and from Nil to Full for initiation, which are described in Table 1. For ignition, the severity classification was deemed as satisfied if any of the conditions was met. For initiation, all of the conditions had to be met.

Table 1. Modes of interaction and classification of their severity.

\begin{tabular}{|c|c|c|c|}
\hline \multicolumn{2}{|c|}{ Ignition } & \multicolumn{2}{c|}{ Initiation } \\
\hline Violent & $\begin{array}{c}\text { Plasma explosion, or violent } \\
\text { movement, or fast event }(<6 \mathrm{~ms})\end{array}$ & Full & $\begin{array}{c}\text { Sample consumed, visible flame, } \\
\text { explosion, change in shape }\end{array}$ \\
\hline Moderate & $\begin{array}{c}\text { Small plasma explosion, or slow } \\
\text { but significant movement }\end{array}$ & $\geq \mathbf{5 0 \%}$ & $\begin{array}{c}\text { As above but } \leq 50 \% \text { of sample } \\
\text { recovered }\end{array}$ \\
\hline Weak & $\begin{array}{c}\text { Some movement, or damage on } \\
\text { inspection by microscope }\end{array}$ & $<50 \%$ & $\begin{array}{c}\text { As above but }>50 \% \text { of sample } \\
\text { recovered }\end{array}$ \\
\hline Nil & No interaction observed & Nil & Sample fully recovered \\
\hline
\end{tabular}


Following the classification of the mode of interaction and the severity, the effect of pulsed laser energy fluence on the three samples (S1, S2 and S3) was measured in terms of these modes and severity and shown in Table 2.

Table 2. Laser ignition / initiation results for pure RDX, CB coated RDX and GNP doped RDX crystals at laser wavelength of $532 \mathrm{~nm}$.

\begin{tabular}{|c|c|c|c|c|c|c|}
\hline $\begin{array}{c}\text { Energy fluence } \\
\left(\mathrm{J} / \mathrm{cm}^{2}\right)\end{array}$ & \multicolumn{2}{|c|}{ Pure RDX } & \multicolumn{2}{c|}{ CB/RDX } & \multicolumn{2}{c|}{ GNP/RDX } \\
\hline & Ignition & Initiation & Ignition & Initiation & Ignition & Initiation \\
\hline 9 & Moderate & - & Violent & - & - & Fully \\
\hline 7.2 & Weak & - & Moderate & - & - & $>50 \%$ \\
\hline 1.4 & Nil & - & Weak & - & - & Fully \\
\hline 0.25 & Nil & - & Nil & - & - & Fully \\
\hline 0.2 & Nil & - & Nil & - & - & $<50 \%$ \\
\hline 0.08 & & & & & - & Fully \\
\hline 0.04 & & & & & - & Fully \\
\hline 0.01 & & & & & Violent & - \\
\hline 0.005 & & & & & Nil & - \\
\hline 0.003 & & & & & Nil & - \\
\hline
\end{tabular}

From the results in Table, it is clear that pure RDX did not initiate, however, it was ignited at high energy fluence, but only at moderate or weak severity. This was due to little or low laser absorption of the sample. CB-coated RDX was not initiated, but ignited with violent severity at high energy fluence. The most violent ignition appears very similar to initiation, but the full sample mass was recovered unconsumed. GNP doped RDX was initiated (fully or partially) at the higher energy fluences than $0.01 \mathrm{~J} / \mathrm{cm}^{2}$. These show that both of CB and GNP optically sensitised RDX, but GNPs doped RDX could be exclusively initiated at low laser pulse energy. Therefore, GNPs doped RDX was the suitable candidate for direct laser initiation.

\subsubsection{Threshold of ignition and initiation}

From the results in Table 2, the thresholds of ignition and initiation for both $\mathrm{CB}$ coated and GNPs doped and pure RDX crystals at $532 \mathrm{~nm}$ laser wavelength are summarised in Table 3, compared with the result using UV $(355 \mathrm{~nm})$ pulsed laser elsewhere [15].

Table 3. Ignition and initiation thresholds for pure, CB coated and GNPs doped $\mathrm{RDX}$ at $532 \mathrm{~nm}$ and comparison with pure RDX at $355 \mathrm{~nm}$. 


\begin{tabular}{|l|c|c|}
\hline \multirow{2}{*}{ Sample } & \multicolumn{2}{|c|}{ Threshold $\left(\mathrm{J} / \mathrm{cm}^{2}\right)$} \\
\cline { 2 - 3 } & Ignition & Initiation \\
\hline Pure RDX at $532 \mathrm{~nm}$ & 7.2 & none \\
\hline Pure RDX at $355 \mathrm{~nm}$ & 1.7 & none \\
\hline CB/RDX at $532 \mathrm{~nm}$ & 1.4 & none \\
\hline GNP/RDX at $532 \mathrm{~nm}$ & 0.01 & 0.04 \\
\hline
\end{tabular}

For mode of ignition, Pure RDX showed no further signs of ignition at lower energy levels and the threshold of ignition was established as $7.2 \mathrm{~J} / \mathrm{cm}^{2}$. Given the weak or little absorption of RDX at the wavelength of $532 \mathrm{~nm}$ compared to its strong absorption at $355 \mathrm{~nm}$, having the molar absorptivity of $1.7 \mathrm{~m}^{2} / \mathrm{mol}$ (or a penetration depth $\sim 70 \mu \mathrm{m}$ ) [3], this result is in line with the threshold of 1.7 $\mathrm{J} / \mathrm{cm}^{2}$ for ignition of RDX crystal by pulsed UV laser at $355 \mathrm{~nm}$. RDX crystal coated with light absorbing CB shows a lower ignition threshold of $1.4 \mathrm{~J} / \mathrm{cm}^{2}$. However, GNPs doped RDX exhibited signs of ignition at as low as energy fluence levels of $0.01 \mathrm{~J} / \mathrm{cm}^{2}$. Compared to the threshold of ignition of pure RDX by $532 \mathrm{~nm}$ and $355 \mathrm{~nm}$ pulsed laser $\left(7.2 \mathrm{~J} / \mathrm{cm}^{2}\right.$ and $1.70 \mathrm{~J} / \mathrm{cm}^{2}$ respectively), this presents a significant reduction in the threshold energy fluence of three and two orders of magnitude respectively. The comparison has concluded that GNP is an effective optical sensitiser which significantly decreases the required laser energy fluence for direct ignition of RDX crystal by a pulsed laser.

For mode of initiation, both pure RDX and CB coated RDX showed no signs of initiation as indicated in Table 3. However, GNP-doped RDX initiated at low energy fluence, resulting in the consumption of all or a significant part of the sample. Its threshold of initiation was established at $0.04 \mathrm{~J} / \mathrm{cm}^{2}$. Notably, some samples of GNP-doped RDX failed to initiate at power levels greater than this threshold. Similarly, as shown in Table 2, the GNP doped RDX sample at 7.2 $\mathrm{J} / \mathrm{cm}^{2}$ and $0.2 \mathrm{~J} / \mathrm{cm}^{2}$ only had $>50 \%$ and $<50 \%$ initiation respectively when the two energy fluences below each of them $\left(1.4\right.$ and $0.25 \mathrm{~J} / \mathrm{cm}^{2}$, and 0.08 and 0.04 $\mathrm{J} / \mathrm{cm}^{2}$ ) had full initiation. It is likely that this was mainly due to sample variation in GNP doping uniformity.

Laser irradiation of energetics such as RDX results in the formation of hotspots at discontinuities, defects and inclusions present in the material through heating by optical absorption. The increase in temperature at the hotspots accelerates exothermic decomposition of the explosive material and eventually achieves a sufficiently high temperature to ignite or initiate the explosive. This is the dominant mechanism responsible for direct laser interaction with explosives [1214]. For GNPs doped RDX crystals, incident laser is strongly absorbed by the GNPs due to the coupling of laser radiation into their localised surface plasmon resonance (SPR), and induces very large enhancement (many orders of magnitudes) of the local electromagnetic field [16-18]. The GNPs heat up and become such hotspots which are confined within the RDX crystals. This mechanism allows internal heating and pressurising in the RDX crystal and efficient utilisation of the laser induced hot GNPs without heat dissipation. For CB-coated RDX crystals, the optical absorption of incident laser mainly occurs 
on the coated surfaces of a RDX crystal and the generated heat may dissipate into the external environment. Therefore, the GNP-doped RDX crystal shows the highest reaction violence and sensitivity to the laser at $532 \mathrm{~nm}$.

\subsubsection{Laser-induced damage}

Crystal samples were recovered from ignition experiments and analysed by microscopy for damage induced by laser irradiation. These images are shown in Figure 8. For pure RDX sample (S1), surface damage caused by moderate ignition as given in Table 2 and 3 was observed on the rear facet of the crystal on the surface opposite to incident surface of the pulse laser irradiation at an energy fluence of $9 \mathrm{~J} / \mathrm{cm}^{2}$. At the rear surface the exiting laser beam size was much smaller than the entering beam at the top surface of the crystal due to the laser focusing along its path. Laser ablation occurred at the rear surface by much higher laser fluence due to the transparency of pure RDX at the $532 \mathrm{~nm}$ wavelength while it didn't at the top surface. Furthermore, surface roughness enhances its optical absorption and may affect the laser ablation. CB-coated RDX sample (S2) exhibited damage on the top surface of the crystal, in line with a small surface ablation and plasma fireball. This was attributed to CB coating on the surface. This sample did not exhibit any damage that extended past the surface layer, as CB wasn't doped inside the crystal and the laser interaction only limited to the CB particles. Importantly, GNP-doped RDX sample (S3) showed significant damage on the laser incident surface and the inside of crystal at even a relatively lower energy fluence $\left(0.2 \mathrm{~J} / \mathrm{cm}^{2}\right)$, with large amounts of materials being consumed in the interaction zone. The crystal cleaved in two and formed tall towers of RDX around the edges of the damage. This may be attributed to laser induced heating on the surface and also within the crystal by efficient absorption of laser energy by GNPs. This also supported the mechanism of direct initiation by pulsed laser of RDX crystals doped with GNPs.

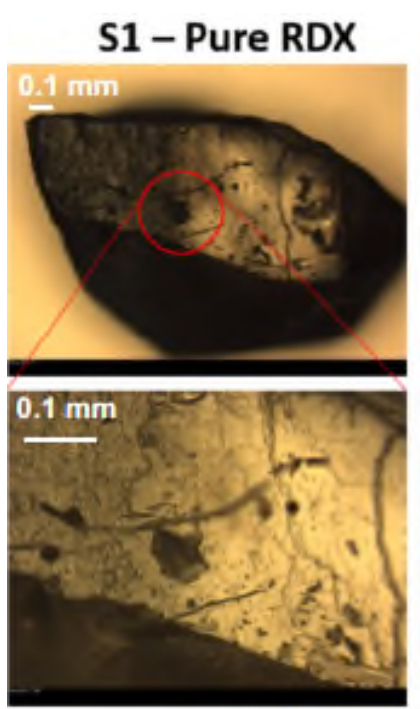

$9 \mathrm{~J} / \mathrm{cm}^{2}$

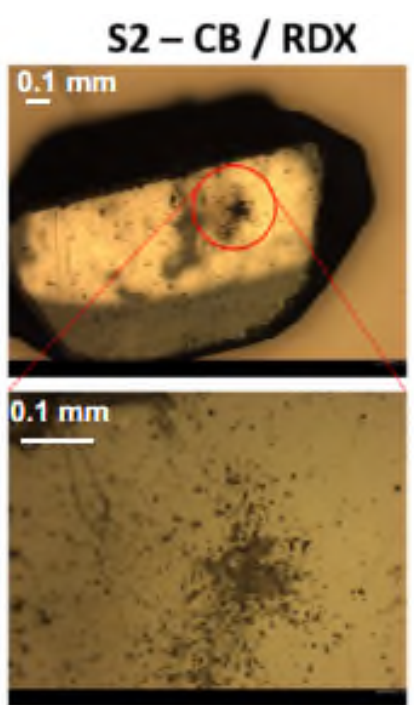

$1.4 \mathrm{~J} / \mathrm{cm}^{2}$

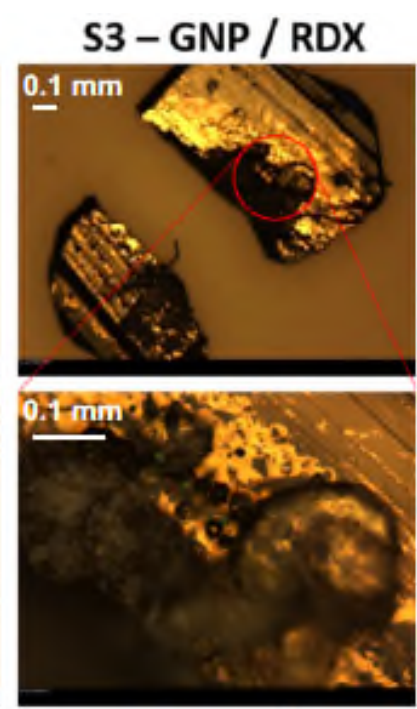

$0.2 \mathrm{~J} / \mathrm{cm}^{2}$

Figure 8. Damage to crystal samples S1, S2 and S3 by 532nm pulsed laser at three different energy fluences. 


\section{Conclusions}

Three modes of interaction were defined between optically sensitised RDX and $532 \mathrm{~nm}$ pulsed laser; nil interaction, ignition (causing micro explosions or plasma fireballs) and initiation (resulting in the sample being fully or partially consumed). Levels of severity of interaction were defined for ignition and initiation. Pure RDX and CB-coated RDX crystals could not be initiated but could be ignited, minimum ignition energy fluence thresholds of $7.2 \mathrm{~J} / \mathrm{cm}^{2}$ and $1.4 \mathrm{~J} / \mathrm{cm}^{2}$ respectively were established. RDX crystals that were optically sensitised with GNPs could be both ignited and initiated, with low laser fluence thresholds of $0.01 \mathrm{~J} / \mathrm{cm}^{2}$ and $0.04 \mathrm{~J} / \mathrm{cm}^{2}$ respectively. The use of GNP as an optical sensitiser through the surface coating and GNP-doping reduced the ignition threshold of RDX by three orders of magnitude and enabled initiation to take place.

\section{Acknowledgements}

The author would like to convey his sincerest gratitude to Dr Mark Carpenter for his assistance in optical microscopy and Dr Ranko Vrcelj for his advices in crystal characterisation.

\section{References}

[1] N.K. Bourne, On the laser ignition and initiation of explosives, Proc. Royal Soc. A 457 (2001) 1401-1426.

[2] X. Fang, S.R. Ahmad, Laser Ignition of an Optically Sensitised Secondary Explosive by a Diode Laser, Cent. Eur. J. Energ. Mater. 13 (2016) 103-115.

[3] P.L. Marinkas, Luminescence of solid cyclic polynitramines, J. Luminescence 15 (1977) 57- 67.

[4] R.A. Isbell, M.Q. Brewster, Optical properties of energetic materials: RDX, HMX, AP,NC/NG, and HTPB, Prop., Explos., Pyro. 23 (1998) 218-224.

[5] Z. Yan, C. Zhang, W. Liu et al, Ultraviolet Laser-induced ignition of RDX single crystal, Sci. Rep. 6 (2016) 20251.

[6] A.L. Ramaswamy, J.E. Field, Laser-induced ignition of single crystals of the secondary explosive cyclotrimethylene trinitramine, J. App. Phys. 79 (1996) 3842-3847.

[7] A. M. Renlund, P.L. Stanton, W.M. Trott, Laser Initiation of Secondary Explosives, 9th International Symposium on Detonation, Portland, Oregon, 1989.

[8] X. Fang, W.G. McLuckie, Laser ignitibility of insensitive secondary explosive 1,1-diamino-2,2-dinitroethene (FOX-7), Journal of Hazardous Materials 285 (2015) 375-382.

[9] X. Fang, M. Sharma, C. Stennett at al, Optical sensitisation of energetic crystals with gold nanoparticles for laser ignition, Combustion and Flame 183 (2017) 15-21.

[10] S. Churchyard, X. Fang, R. Vrcelj, Doping RDX crystals with metal nanoparticles to increase laser ignitability, Optics and Laser Technology 113 (2019) 281-288.

[11] N.N. De, N.R. Cummock, I.E. Gunduz et al, Photoflash and laser ignition of 
select high-nitrogen materials, Combustion and Flame 167 (2016) 207-217.

[12] J. A. Brown, D.A. LaBarbera, M.A. Zikry, Laser interaction effects of electromagnetic absorption and micro structural defects on hot-spot formation in RDX-PCTFE energetic aggregate, Modell. Simul. Mater. Sci. Eng. 22 (2014) 55013.

[13] M.W. Chen, S. You, K.S. Suslick et al, Hot spot generation in energetic materials created by long-wavelength infrared radiation, Appl. Phys. Lett. 104 (2014) 061907.

[14] M.W. Chen, S. You, K.S. Suslick et al, Hot spots in energetic materials generated by infrared and ultrasound, detected by thermal imaging microscopy, Rev. Sci. Instrum. 85 (2014) 023705.

[15] Z. Yan, W. Liu, Y. Jiang et al, Laser initiation of RDX crystal slice under ultraviolet and near-infrared irradiations, Combustion and Flame 190 (2018) 112-118.

[16] Y. Cui, Y. He, Y. Jin et al, Plasmonic and metamaterial structures as electromagnetic absorbers, Laser and Photonics Reviews 8 (2014) 495-520.

[17] M. Schnell, P. Alonso-Gonzalez, L. Arzubiaga et al, Nanofocusing of midinfrared energy with tapered transmission lines, Nat. Photonics 3 (2011) 283287.

[18] H. Cang, A. Labno, C. Lu et al, Probing the electromagnetic field of a 15nanometre hotspot by single molecule imaging, Nature 469 (2011) 385-388. 